\title{
Title: Spatiotemporal patterns of current source density in the prefrontal cortex of a behaving monkey
}

Authors: Kazuhiro Sakamoto ${ }^{\mathrm{a} *}$, Norihiko Kawaguchi ${ }^{\mathrm{b}}$, Kohei Yagi ${ }^{\mathrm{b}}$, and Hajime Mushiake $^{\mathrm{b}, \mathrm{c}}$.

\section{Affiliations:}

${ }^{a}$ Research Institute of Electrical Communication, Tohoku University, 2-1-1 Katahira, Aoba-ku, Sendai 980-8577, JAPAN.

${ }^{\mathrm{b}}$ Department of Physiology, Tohoku University School of Medicine, 2-1 Seiryo-cho, Aoba-ku, Sendai 980-8575, JAPAN.

${ }^{c}$ The Core Research for Evolutional Science and Technology Program (CREST), JST, Goban-cho, Chiyoda-ku, Tokyo, 102-0076, JAPAN.

* To whom correspondence should be addressed. E-mail: sakamoto@riec.tohoku.ac.jp 


\begin{abstract}
One of the fundamental missions of neuroscience is to explore the input and output properties of neuronal networks to reveal their functional significance. However, it is technically difficult to examine synaptic inputs into neuronal circuits in behaving animals. Here, we conducted current source density (CSD) analysis on local field potentials (LFPs) recorded simultaneously using a multi-contact electrode in the prefrontal cortex (PFC) of a behaving monkey. We observed current sink task-dependent spatiotemporal patterns considered to reflect the synaptic input to neurons adjacent to the recording site. Specifically, the inferior convex current sink in the PFC was dominant during the delay period, whereas the current sink was prominent in the principal sulcus during the sample cue and test cue periods. Surprisingly, sulcus current sink patterns were spatially periodic, which corresponds to the columnar structure suggested by previous anatomical studies. The approaches used in the current study will help to elucidate how the PFC network performs executive functions according to its synaptic input.
\end{abstract}




\section{Introduction}

It is important to explore the input-output structures of cortical circuits to clarify their functional roles. In particular, it is crucial to elucidate the executive functions associated with the prefrontal cortex (PFC) (Fuster, 2000, 2008; Passingham \& Wise, 2012). That is, the PFC is responsible for determining actions or action plans according to sensory and mnemonic inputs rather than merely responding to them. Correspondingly, recent physiological and theoretical studies have revealed that the output properties of neurons in PFC circuits dynamically change depending on their supposed inputs (Katori, et al., 2011; Machens, Romo \& Brody, 2005; Sakamoto et al., 2008, 2013). However, signals obtained from conventional single-unit recordings are spikes that reflect the output of neurons or neuronal circuits, and signals that directly reflect the inputs to them are unclear. Here, we analyzed local field potentials (LFPs) recorded from the PFC of a behaving monkey and obtained current source density (CSD) spatiotemporal patterns thought to reflect synaptic input current (Freeman \& Nicholsnon, 1975; Mitzdorf, 1985; Nicholson \& Freeman, 1975). 


\section{Methods}

\subsection{Subjects and apparatus}

Experiments were performed on one male monkey (Macaca fuscata; $9.0 \mathrm{~kg}$ ). All experimental protocols were approved by the Animal Care and Use Committee of Tohoku University. Furthermore, all animal protocols conformed with the National Institutes of Health $(\mathrm{NIH})$ guidelines for the care and use of laboratory animals and to recommendations of the Weatherall Report. The animal was seated in a primate chair with his head restrained and oriented toward a computer monitor.

\subsection{Behavioral task}

The monkey was trained to perform a shape manipulation task that required step-by-step movements with manipulanda. The goal was to fit the test shape to the sample shape (Fig. 1). In each trial, a single shape was randomly selected from a set of display shapes. A set of four shapes was included in the recording sessions, whereas various shape types were used in the training sessions.

After a fixation spot appeared on the screen, a sample shape was displayed for $1 \pm 0.2$ $\mathrm{s}$ (sample cue). Following a $1 \pm 0.2 \mathrm{~s}$ delay period (delay), a test shape that was homothetic to the sample shape but transformed (i.e., expanded/contracted and rotated) was displayed for $1 \pm 0.2 \mathrm{~s}$ (test cue). Thereafter, the color of the fixation point was changed, which served as a go signal to initiate the first-step movement (first go). The animal was required to execute a movement within a specific time window and to wait until the second go signal (second go). At each go signal, the animal was allowed to make a single, one-handed movement. He was also permitted to perform any number of steps as long as a movement was executed within the time window. When the test shape was successfully transformed to fit the sample shape, the program automatically stopped the trial and the animal was rewarded with an isotonic drink. 
Cursor movement was linked to the movements of two manipulanda installed in the chair, which were operated with the right or left wrist. Left-hand supination and pronation and right-hand supination and pronation controlled the expansion (double the area), contraction (half the area), rightward rotation (-45 deg.) and leftward rotation (45 deg.) of the test shape, respectively.

To dissociate the movements of the arms and the cursor, we trained the monkey to perform the task with two different arm-cursor assignments. In the first cursor assignment, left-hand supination, left-hand pronation, right-hand supination, and right-hand pronation were assigned to expansion, contraction, rightward rotation, and leftward rotation, respectively. In the second assignment, left-hand supination, left-hand pronation, right-hand supination, and right-hand pronation were assigned to leftward rotation, rightward rotation, contraction, and expansion, respectively. The assignment was changed every 68 trials without any instructions. In the first half of the 68 -trial block, the contour of the sample cue was displayed until the first go signal was delivered (visually guided task), whereas the contour was not displayed in the latter half (memory-guided task). The minimum-step success rate was 89\% (Sakamoto, Kawaguchi, \& Mushiake, 2012).

\subsection{Surgery and electrophysiological recording}

After the behavioral training, a polyacetal resin recording chamber was attached to the monkey's skull under aseptic conditions. During surgery, the animal was anesthetized with ketamine hydrochloride (10 $\mathrm{mg} / \mathrm{kg}$, intramuscularly) and pentobarbital sodium (30 mg/kg, intramuscularly). Following surgery, cortical sulci were identified using a magnetic resonance imaging (MRI) scanner (OPART 3D-System; Toshiba). Cortical sulci were also confirmed by mapping using single-unit activities recorded using conventional metal electrodes. Furthermore, the frontal eye 
field (FEF) was identified using intracortical microstimulation (ICMS; Bruce et al., 1985). This procedure enabled us to record LFP signals from the PFC, the area rostral to the sites at which ICMS current $<80 \mu \mathrm{A}$ did not evoke saccades including the banks of the principal sulcus and the adjacent cortical convexity.

Electrophysiological recordings were conducted using linear-array multi-contact electrodes (U-Probe; Plexon) containing 15 recording contacts (impedance, 0.3-1.3 M $\Omega$ at $1 \mathrm{kHz}$ ) with an intercontact spacing of $200 \mu \mathrm{m}$ to obtain LFPs. We used a guide needle to help the electrode penetrate because a linear-array multi-contact electrode was not able to pass through the dura matter by itself. Specifically, the guide needle was stopped right before it passed through the dura matter. Then, only the electrode was inserted into the cortex perpendicular to its surface. The position of the electrode was strictly determined. The electrode was carefully lowered, and the multi-unit activity initially recorded by the bottommost contact (ch. 15) was transferred to ch. 14 by moving the electrode $200 \mu \mathrm{m}$. The transferred unit was readily identified as the initial unit by its waveform and monitored sound. We repeated this procedure until the unit was recorded through the topmost contact (ch. 1). This procedure guaranteed that multi-unit activities were recorded from almost all the contacts. Consequently, the bottommost contact (ch. 15) was confirmed to be located within the gray matter in all recording sessions. The reference electrode was placed on the dura mater surface. The electrode was driven by a hydraulic microdrive manipulator (Narishige). Signals from the electrode were collected using a data acquisition system (Neuralynx). LFP was obtained by band-pass filtering the raw signal from $0.1 \mathrm{~Hz}$ to $475 \mathrm{~Hz}$ and digitizing the signal at $1.24 \mathrm{kHz}$. Spikes were always monitored at the same time.

\subsection{Data analysis}


Only the data obtained from correct minimal two-step trials were included in further analyses. LFP signals were averaged across trials. CSD was calculated from LFPs by numerical differentiation to approximate the second-order spatial derivative of the voltage recorded at each recording contact. CSD analysis provided a physiological index of the location, direction, and density of the transmembrane current flow of the corresponding depths of the cortex (Freeman \& Nicholsnon, 1975; Mitzdorf, 1985; Nicholson \& Freeman, 1975). CSD at the n-th contact, $D_{n}$, was calculated as follows:

$D_{n}=-\sigma[\varphi(\mathrm{n}+1)+\varphi(\mathrm{n}-1)-2 \varphi(\mathrm{n})] / \Delta^{2}$,

where $\sigma$ is the tissue conductivity assumed to be constant $(0.3 \mathrm{~S} / \mathrm{m}), \varphi(\mathrm{n})$ is the LFP signal at the $\mathrm{n}$-th contact of the electrode, and $\Delta$ is the spacing between the neighboring electrode contacts $(200 \mu \mathrm{m})$. Negative and positive $D_{n}$ indicate the current sink and current source at the n-th contact, respectively. CSD waveforms were smoothed by sliding an averaging window of $50 \mathrm{~ms}$. The presence of a peak and trough, the source and sink of current, respectively, was statistically tested using a bootstrapping method. We defined the reference period as the time from $-1,000$ to $-500 \mathrm{~ms}$ of the sample cue onset for each data set because no salient current sinks or sources were seen in any data set during this period. We calculated all possible quasi-CSD values by shuffling the channel number of the electrode at each time point, then we obtained lower and upper significance levels of $P=0.05$ and $P=0.95$, respectively. Only epochs exhibiting significant sinks or sources in consecutive time steps for more than $50 \mathrm{~ms}$ were regarded as significant. 


\section{Results}

\subsection{Dissociation of convex and sulcus}

We recorded LFP signals from 10 sites on the ventral side of the PFC principal sulcus (Fig. 2A). Each data set included about 140 correct trials with a minimum of two steps (i.e., entire task variation). Meanwhile, we stably recorded neuronal data. Data were analyzed for the preparatory period of the task.

The 10 recording sites were clearly divided into two groups: the convex and sulcus groups. Specifically, four recording sites, $(23.0,35.0),(23.0,36.0),(24.0,35.0)$, and $(24.0,36.0)$, in the medio-lateral (ML) and antero-posterior (AP) coordinates were from the lateral bank of the principal sulcus (sulcus group: black circles in Fig. 2A), whereas the remaining six sites were from the inferior convex (convex group: gray circles in Fig. 2A). The MRI image of the AP 35.0 plane clearly showed the principal sulcus (Fig. 2B), which completely disappeared in the AP 34.0 image that was only 1.0 $\mathrm{mm}$ posterior to it (Fig. 2C). These images demonstrate that penetrations in the sulcus group were almost parallel to the sulcus and that those in the convex group were nearly perpendicular to the cortical surface. These were also confirmed by the physiological mapping of spike activities using conventional metal electrodes conducted beforehand.

Examples of CSD waveforms in the two groups during the reference period (see Methods) are presented in Figures 2D and E. In this period, we did not observe any salient current sinks or sources. However, the variability of these baseline waveforms differed between the two groups. That is, the CSD waveforms of the sulcus group were noisy. This inspection was statistically confirmed by comparing the standard deviations of the two groups (Mann-Whitney $U$ test, $P<0.01$; Fig. 2F). All values of the sulcus group were greater than were those of the convex group.

\subsection{Spatiotemporal pattern of current sink}


CSD data on current sink reflected excitatory synaptic input to neurons at the recording site (Buzsáki, Anastassiou, \& Koch, 2012; Einevoll, Kayser, Logothetis, \& Panzeri, 2013). In the following section, we examine the spatiotemporal patterns of current sink, namely, the recording sites, the depth and the task phase at which significant sinks were observed. In particular, we focus on the preparatory period during which information about the sample and test cues should be input to the PFC.

Figure 3A depicts an example of CSD patterns from the convex group, the same data as those in Figure 2D. In this example, a significant current sink at ch. 10 during the sample cue period was followed by a strong sink at ch. 9 , accompanied by a weak one at ch. 13, during the delay period. After that, a sink appeared at ch. 12 at around the first go signal. As a whole, significant sinks appeared in this convex site only at deeper contacts located at about $2 \mathrm{~mm}$ or more from the surface of the cortex.

In contrast, the sulcus group example illustrated in Figures $3 \mathrm{~B}$ and $2 \mathrm{E}$ exhibited strong current sinks even at upper contacts. In the sample cue period, significant sinks were detected at ch. 12 and 14, whereas sinks with the same timing were also seen at upper contacts. As the delay period approached, significant and non-significant sinks were observed at ch. 5 and 13, respectively. Sinks were not apparent in the main part of the delay period. In contrast, right before the test cue period, a strong sink emerged at $\mathrm{ch}$. 13, and this was followed by strong sinks at ch. 5, 9, and 13 during the test cue period. The strong sinks in this period seemed to be regularly associated with the four points of contact (i.e., $800-\mu \mathrm{m}$ intervals).

The general tendencies shown in Figure 3 were apparent in the spatiotemporal patterns of the significant sinks obtained from the entire data set (Fig. 4). That is, almost all of the significant sinks in the convex group were observed in contacts below ch. 9, more than $1,600 \mu \mathrm{m}$ from the cortical surface $(24 / 25, P<0.001$, binominal test). In contrast, several significant sinks were detected in the upper contacts of the sulcus 
group compared with the convex group $(9 / 10, P<0.01$, binominal test). The task-dependent current sink pattern revealed that the sulcus group exhibited a substantially more significant sink during the sample cue period than during the other periods $(7 / 24, P<0.05$, binominal test). The number of significant sinks was also greater in the sulcus group than in the convex group during the sample cue period $(7 / 8$, $P<0.01$, binominal test). In contrast, the convex group exhibited a more significant sink in the delay period than did the sulcus group, although the difference was not statistically significant $(7 / 8, P=0.11$, binominal test). Furthermore, multiple recording sites in the sulcus groups exhibited a "three-stripe" sink pattern during the test period, each separated by several hundred micrometers. 


\section{Discussion}

In this study, we simultaneously recorded multiple LFP signals from the PFC of a behaving monkey. Through CSD analysis, we defined the current sink positions that reflected synaptic input at each recording site and obtained their spatiotemporal patterns. We recognized clear distinctions in the CSD signals between recording sites located in the inferior convex and those located in the principal sulcus. In the convex group, the electrode penetrations were estimated to be nearly perpendicular to the layer structure of the cortex, the baseline of CSD signals had smaller variances, and significant current sinks were detected at the lower contacts. In the sulcus group, the penetrations were expected to be almost parallel to the layer structure, the CSD baselines fluctuated, and significant sinks were observed not only at lower contacts but also at upper ones. It should be noted that the sink patterns in the sulcus groups seemed periodic with about $800-\mu \mathrm{m}$ intervals. The task phases in which current sinks were prominent differed in these two groups. The sulcus group exhibited many sinks in the sample cue and test cue periods but not in the delay period, during which many sinks were seen in the convex group.

Division of the 10 recording sites into two groups (i.e., sulcus and convex groups) was highly reliable. Note that the examples of both groups presented in Figures 2D, 2E, and 3 were recorded from sites separated by only $1 \mathrm{~mm}$, but they exhibited quite different characteristics, as mentioned above. This discrepancy is consistent with the results of physiological mapping undertaken before the main recording session. That is, conventional unit recordings with metal electrodes revealed that the principal sulcus terminates between AP 34.0 and 35.0 positions. This was also confirmed by MRI imaging, as illustrated in Figures 2B and C.

CSD baseline variability was much lower in the convex group than in the sulcus group. This result indicated that the LFP signals are highly homogeneous or correlated 
in the vertical direction of the cortical layer structure. This also supports the conventional idea that the bipolar structure emerges from the spatial separation of current sink and source along the dendritic structure in pyramidal cells across cortical layers, which likely contributes to LFP generation (for reviews, Buzsáki, Anastassiou, \& Koch, 2012; Einevoll, Kayser, Logothetis, \& Panzeri, 2013).

Estimations of the current sink position in the vertical direction were also very reliable. The multi-contact electrode was inserted into the cortex with great care, minimizing tissue damage. We did so according to the following protocol. When we encountered the first spiking neuron, we moved the electrode so that the neuron was recorded from the top contact, ch. 1. After that, we waited before recording from the behaving animal to obtain stable recordings. The accuracy of this procedure was presumably reflected in the good reproducibility of the results. The fact that the current sink positions in the convex group were almost limited to the contacts below ch. 9 is a good example.

The current-sink positions of the convex group seemed to be separated into two bands, around ch. 9-10 and around ch. 14. As seen in Figure 4, these two bands sometimes appeared simultaneously. Considering that the ch. 9-10 and ch. 14 bands were located at around $1,700 \mu \mathrm{m}$ and 2,600 $\mu \mathrm{m}$ of depth, respectively, the two bands may correspond to the granular and the infragranular layers, respectively (Kajikawa, \& Schroeder, 2011; Takeuchi, Hirabayashi, Tamura, \& Miyashita, 2011). The granular and the infragranular layers are hypothesized to be "feed-forward" and "feedback" inputs, respectively, in reciprocal connections between cortical areas constituting a hierarchical structure (Fellman \& Van Essen, 1991). Thus, future studies should examine the tendency shown in Figure 4: that sinks were observed more frequently in the ch.-14 band than in the ch. 9-10 band.

Unlike the convex group, the sulcus group exhibited significant sinks at the upper 
contacts. Surprisingly, a current sink periodic pattern with about $800-\mu \mathrm{m}$ intervals was observed, as illustrated in Figure 3B. Among the recording sites in the sulcus group, the site $(23.0,36.0)$ was rather silent. However, this periodic structure was recognizable even in this site (data not shown). Weak current sinks in this site might have been caused by the sparseness of contacts. That is, unfortunately, each contact might have been located between a current sink and source and thus failed to detect a strong current sink or source. This periodic structure of several hundred micrometers seems to correspond quite well to the columnar structure of afferents from contralateral PFC and ipsilateral parietal cortex reported by anatomical studies conducted a few decades ago (Goldman \& Nauta, 1977; Goldman-Rakic \& Schwartz, 1982). Our results can be regarded as the first report that directly suggests a columnar structure in the PFC using a physiological approach. The columnar structure of the PFC allows various inputs to produce different LFP gradient patterns. The LFP gradient, including the phase of LFP oscillation, is likely related to the modulation of firing activity (Murthy \& Fetz, 1992; Lee et al., 2005); thus, such variable modulation may contribute to the emergent properties of the PFC beyond executive function.

In this study, we targeted the subpart of the PFC ventral to the principal sulcus, as our shape manipulation task required working memory about visual shapes (Wilson, O’Scalaidhe \& Goldman-Rakic, 1993). However, this subpart may be separated into different cortical areas when these are defined anatomically and histologically, namely the lateral bank of the principal sulcus and inferior convexity (Passingham \& Wise, 2012; Paxinos, Huang, \& Toga, 2000). Our results show that current sinks were dominant during the delay period in the convex group, whereas they were prominent during the sample cue and the test cue periods in the sulcus group. This discrepancy most probably reflects the difference in neuronal connectivity to other areas (Petrides \& Pandya, 1994; Passingham \& Wise, 2012). 
Our use of this modern physiological technique, coupled with the application of multi-contact electrodes and CSD analysis, constitutes a promising approach. This technique enables examination of fine cortical input structures (i.e., from layer structures to columnar structures). Combined analysis of the spike activities reflecting the output of the neuronal circuits of interest can reveal the functional roles of the circuits in greater detail. In terms of the PFC, the executive function and other creative aspects of intelligence for which the PFC is supposedly responsible should require emergent properties in its neuronal circuit. We must determine what emerges between input and output to understand these properties. The new technique mentioned above will make this possible. 


\section{Acknowledgements}

This research was supported by a Grant-in-Aid for Scientific Research (C) (\#22500283, \#26350991) by the Japan Society for the Promotion of Science; a Grant-in-Aid for Scientific Research on Innovative Areas "The study on the neural dynamics for understanding communication in terms of complex hetero systems (number 4103)" (\#22120504, \#24120703) and "Elucidation of the neural computation for prediction and decision making (number 4303)" (\#26120703) of the Ministry of Education, Culture, Sports, Science, and Technology of Japan; Creative Interdisciplinary Research Program in Frontier Research Institute for Interdisciplinary Sciences of Tohoku University; the Japan Science and Technology Agency (Exploratory Research for Advanced Technology and Core Research for Evolutional Science and Technology). We thank T. Suenaga, K. Sato, H. Watanabe, M. Kurama, and M.Takahashi for technical assistance. 


\section{References}

Bruce, C. J., Goldberg, M. E., Bushnell, M. C., \& Stanton, G. B. (1985). Primate frontal eye fields. II. Physiological and anatomical correlates of electrically evoked eye movements. Journal of Neurophysiology, 54, 714-734.

Buzsáki, G., Anastassiou, C. A., \& Koch, (2012). The origin of extracellular fields and currents - EEG, ECoG, LFP and spikes. Nature Review Neuroscience, 13, 407-420.

Einevoll, G. T., Kayser, C., Logothetis, N. K., \& Panzeri, S. (2013). Modelling and analysis of local field potentials for studying the function of cortical circuits. Nature Review Neuroscience, 14, 770-785.

Fellman, D. J., \& Van Essen, D. C. (1991). Distributed hierarchcal processing in the primate cerebral cortex. Cerebral Cortex, 1, 1-47.

Freeman, J. A., \& Nicholson, C. (1975). Experimental optimization of current source density technique for anuran cerebellum. Journal of Neurophysiology, 38, 369-382.

Fuster, J. M. (2000). Executive frontal functions. Experimental Brain Research, 133, $66-70$.

Fuster, J. M. (2008). The prefrontal cortex. Academic Press.

Goldman, P. S., \& Nauta, W. J. (1977). Columnar distribution of cortico-cortical fibers in the frontal association, limbic, and motor cortex of the developing rhesus monkey. Brain Research 122, 393-413.

Goldman-Rakic, P. S., \& Schwartz, M. L. (1982). Interdigitation of contralateral and ipsilateral columnar projections to frontal association cortex in primates. Science, $216,755-757$.

Kajikawa, Y., \& Schroeder, C. E. (2011). How local is the local field potential? Neuron, $72,847-858$.

Katori, Y., Sakamoto, K., Saito, N., Tanji, J., Mushiake, H., \& Aihara, K. (2011). 
Representational switching by dynamical reorganization of attractor structure in a network model of the prefrontal cortex. PLoS Computational Biology, 7, e1002266.

Lee, H., Simpson, G. V., Logothetis, N. K., \& Rainer, G. (2005). Phase locking of single neuron activity to theta oscillations during working memory in monkey extrastriate visual cortex. Neuron, 45, 147-156.

Machens, C. K., Romo, R., \& Brody, C. D. (2005). Flexible control of mutual inhibition: a neural model of two-interval discrimination. Science, 307, 1121-1125.

Mitzdorf, U. (1985). Current source density method and application in cat cerebral cortex: investigation of evoked potentials and EEG phenomena. Physiological Review, 65, 37-100.

Murthy, V. N., \& Fetz, E. E. (1992). Coherent 25- to $35-\mathrm{Hz}$ oscillations in the sensorimotor cortex of awake behaving monkeys. Proceedings of the National Academy of Sciences of the United States of America, 89, 5670-5674.

Nicholson, C., \& Freeman, J. A. (1975). Theory of current source density analysis and determination of conductivity tensor for anuran cerebellum. Journal of Neurophysiology, 38, 356-368.

Passingham, R. E., \& Wise, S. P. (2012). The neurobiology of the prefrontal cortex: Anatomy, Evolution, and the Origin of Insight. Oxford.

Paxinos, G., Huang, X-F., \& Toga, A. W. (2000). The rhesus monkey brain in stereotaxic coodinates. Academic Press.

Petrides, M., \& Pandya, D.N. (1994). Comparative architectonic analysis of the human and the macaque frontal cortex. In F. Boller, \& J. Grafman (Eds.), Handbook of Neuropsychology (pp. 17-58). Amsterdam: Elsevier.

Sakamoto, K., Katori, Y., Saito, N., Yoshida, S., Aihara, K., \& Mushiake, H. (2013). Increased firing irregularity as an emergent property of neural-state transition in monkey prefrontal cortex. PlosONE, 8, e80906. 
Sakamoto, K., Kawaguchi, N., \& Mushaike H. (2012). Is advance planning of sequential movements reflected in the behavior of monkeys? Annuals of Japan Neural Network Society, 22, 3-19.

Sakamoto, K., Mushiake, H., Saito, N., Aihara, K., Yano, M., \& Tanji, J. (2008). Discharge synchrony during the transition of behavioral goal representations encoded by discharge rates of prefrontal neurons. Cerebral Cortex, 18, 2036-2045.

Takeuchi, D., Hirabayashi, T., Tamura, K., \& Miyashita, Y. (2011). Reversal of interlaminar signal between sensory and memory processing in monkey temporal cortex. Science, 331, 1443-1447.

Wilson, F. A. W., O’ Scalaidhe, S. P., \& Goldman-Rakic, P. S. (1993). Dissociation of object and spatial processing domains in primate prefrontal cortex. Science, 260, 1955-1958. 


\section{Figure legends}

Figure 1.

Temporal sequence of events in the shape manipulation task. The behavioral sequence is depicted from the top right to the bottom left. Each panel represents a display presented to the animal during each task epoch. The central dot denotes the fixation point by which movement initiation was signaled.

\section{Figure 2.}

Recording sites. A. 10 recording sites from the prefrontal cortex. Gray circles denote recording sites from the inferior convex. Black circles, from the lateral bank of the principal sulcus. B, C. The coronal images of MRI (T2) at anterior-posterior (AP) 35.0 and 34.0, respectively. Note that the principal sulcus in the AP 35.0 image disappears in the AP 34.0 image. D, E. Examples of the waveforms of trial-averaged current source density (CSD) obtained for each contact during the reference period (from -1,000 to $-500 \mathrm{~ms}$ from sample onset) from the inferior convex and the principal sulcus, respectively. The electrode positions are indicated in $\mathbf{A}, \mathbf{B}$, and $\mathbf{C}$. Vertical and horizontal scale bars, $5 \mu \mathrm{A} / \mathrm{mm}^{3}$.and $100 \mathrm{~ms}$, respectively. F. Difference in CSD variability between the convex and sulcus. Averaged CSD standard deviations are shown. Asterisk, $P<0.01$.

\section{Figure 3.}

Examples of trial-averaged CSD patterns. Each raw value indicates the CSD waveforms for each contact of the electrode schematized in the right. Waveforms were aligned to the sample- and test-cue onsets (black triangles). A. An example from the convex at $(\mathrm{ML}, \mathrm{AP})=(23.0,34.0)$. B. An example from the principal sulcus at $(23.0,35.0) . \mathrm{M}$, L, A and P denote medial, lateral, anterior, and posterior, respectively. 


\section{Figure 4.}

The spatiotemporal pattern of significant current sink. The position of each pentagonal shape represents the position of a recorded site. Vertical positions of the black bars within the pentagon represent the vertical positions of significant current sink. Dotted lines are the border between the sulcus and convex. Peri-sample Onset, Sample Cue, Peri-delay Start, and Delay, -400 to $100 \mathrm{~ms}, 100$ to $600 \mathrm{~ms}, 600$ to $1,100 \mathrm{~ms}$, and 1,100 to $1,600 \mathrm{~ms}$ of the sample cue onset, respectively. Peri-test Onset, Test Cue, and Peri-1st Go, -400 to $100 \mathrm{~ms}, 100$ to $600 \mathrm{~ms}$, and 600 to $1,100 \mathrm{~ms}$ of the test cue onset, respectively. 


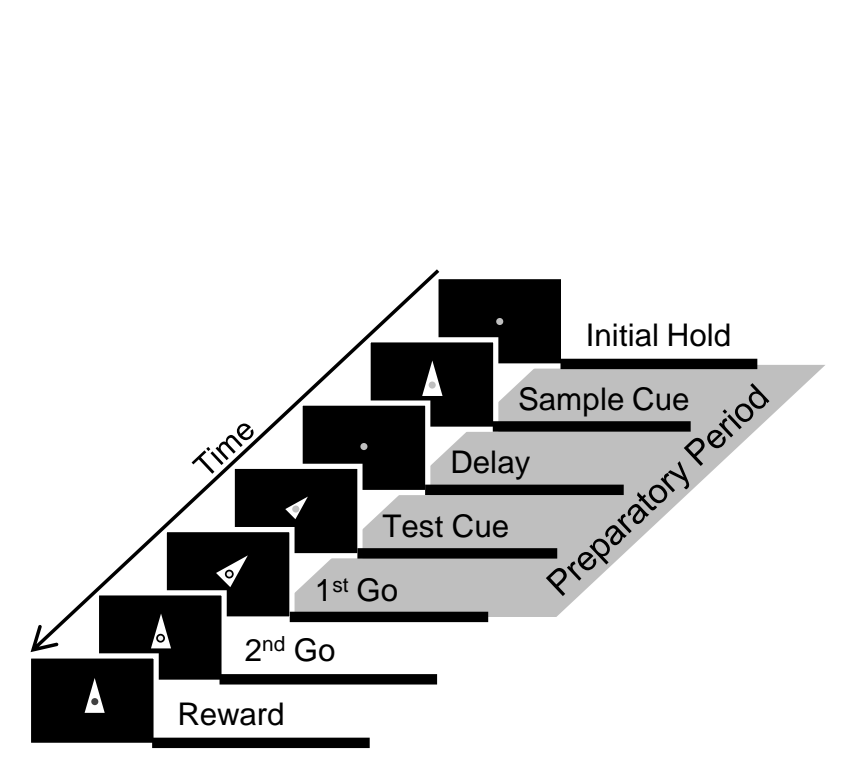

\section{Fig.1}

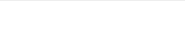



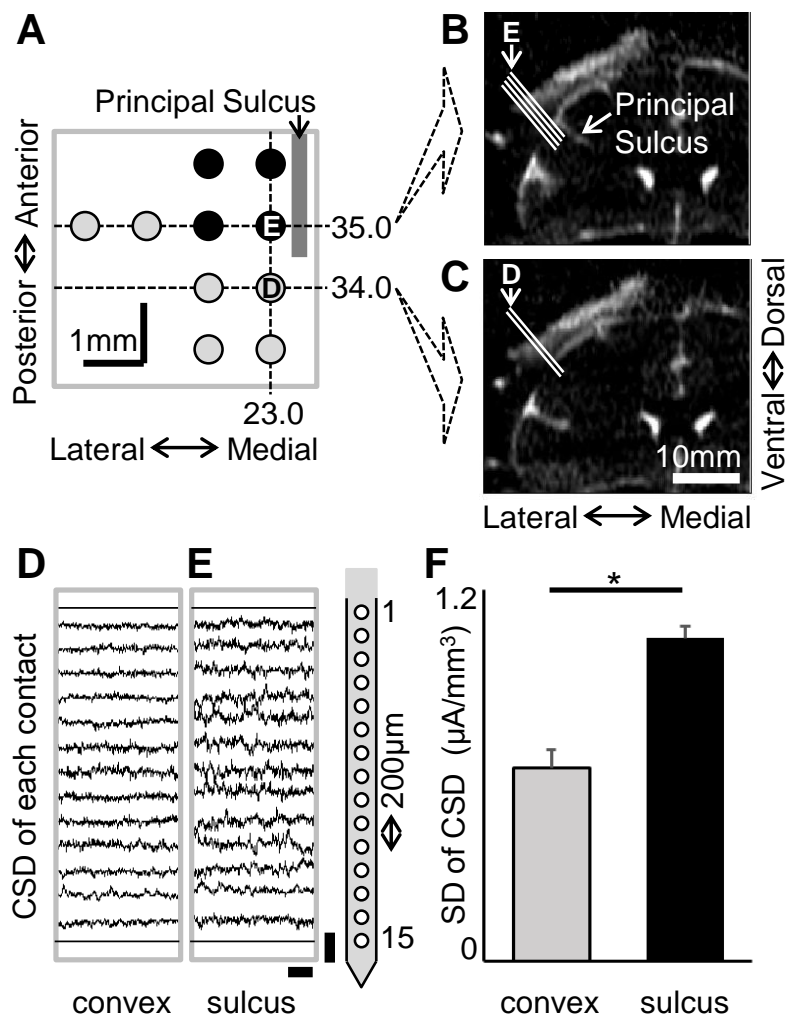

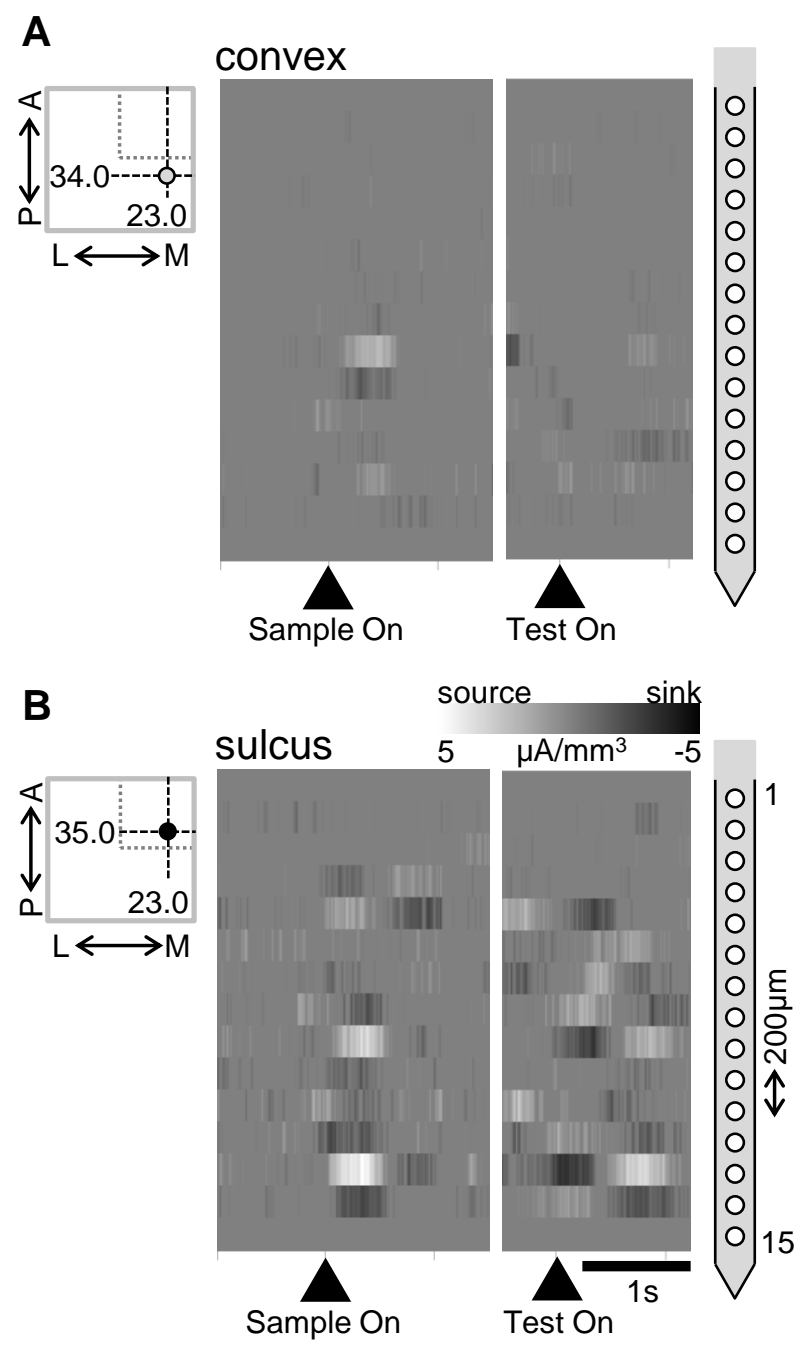

\section{Fig.3}




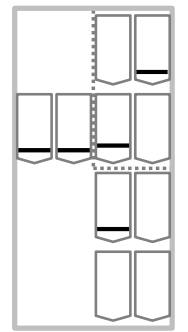

Peri-Sample Onset

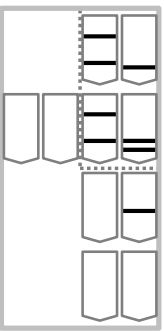

Sample Cue

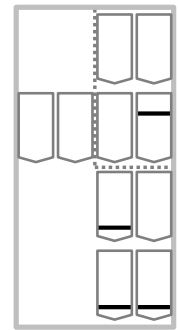

Peri-Delay Start

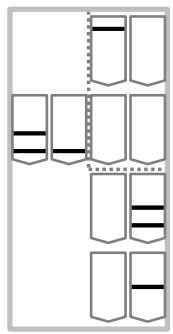

Delay

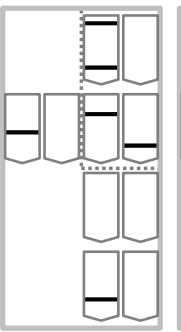

Peri-Test Onset

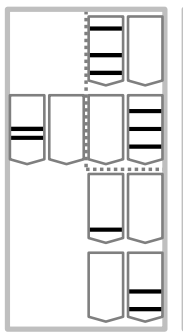

Test

Cue

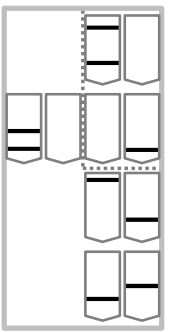

Electrode position

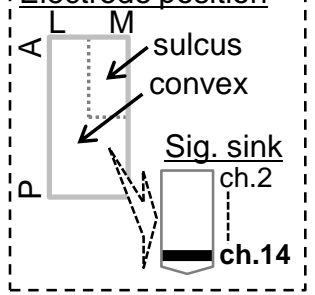

Peri-

$1^{\text {st }} \mathrm{Go}$

\section{Fig.4}

